\title{
Currents structure in the scrape-off layer of a tokamak in a quiescent state
}

\author{
V. Rozhansky ${ }^{1}$, E. Kaveeva ${ }^{1}$, I. Senichenkov ${ }^{1}$, D. Sorokina ${ }^{1}$, E. Vekshina ${ }^{1}$, D. Coster ${ }^{2}$, P. \\ McCarthy ${ }^{3}$, N. Khromov ${ }^{4}$ and the ASDEX Upgrade team \\ ${ }^{1}$ Peter the Great St.Petersburg Polytechnic University, Polytekhnicheskaya 29, 195251 St.Petersburg, Russia \\ ${ }^{2}$ Max-Planck Institut für Plasmaphysik, EURATOM Association, D-85748 Garching, Germany \\ ${ }^{3}$ Department of Physics, University College Cork, Cork, Ireland \\ ${ }^{4}$ Ioffe Institute, Polytechnicheskaya 26, St.Petersburg, Russia \\ rozhansky@mail.ru
}

\begin{abstract}
Currents structure in the scrape-off layer (SOL) of a tokamak is analyzed. It is demonstrated that poloidal currents measured in the experiments are a combination of several current types of different physical nature. Besides known Pfirsch-Schlüter (PS) currents and thermoelectric currents, so-called plate closing currents (PCC) flowing to/from the divertor plates are also analyzed. The latter close radial currents in the SOL and below/above the $X$ point in the SOL and private flux region (PFR). In particular, current flowing to the outer plate in the private flux region, opposite to thermoelectric current is predicted for the standard singlenull configuration and favorable direction of $\nabla B$ drift. In addition, a pair of currents to and away from the outer plate should flow. In the single-null configuration they are often masked by a larger thermoelectric current, however for the connected double null (CDN) case, where thermoelectric current is strongly reduced due to smaller temperature asymmetry, these currents dominate. The suggested physical model is supported by results of simulations performed with SOLPS-ITER transport code. Simulations were done for ASDEX Upgrade (AUG), L and H-modes, single-null configurations, and for GLOBUS-M H-modes, both disconnected and connected double null configurations. Results of the simulations are compared with probe measurements for AUG and Globus-M tokamaks, and reasonable agreement has been found. The role of parallel currents in the formation of the potential maximum/minimum in the vicinity of $X$-point for strongly detached regimes is also analyzed.
\end{abstract}

\section{Introduction}

Current structure in the edge of a tokamak is quite complicated. Besides widely known parallel PS currents [1] and thermoelectric currents [2], there are other currents flowing through the divertor plates, which close radial currents in the plasma volume [1], [3]-[5]. These currents 
might be called plate closing currents (PCC). Below we analyze the structure of all currents in the edge plasma both in the SOL and divertor regions, including currents flowing to the divertor plates. Currents in the SOL determine potential profiles, and, in particular, potential distribution along the divertor plates. The latter can have a significant impact on the sheath heat transmission factor and hence on the electron temperature and the density near the divertor plates and particle flux to the divertor plates. In the detached regimes, where strong parallel currents require strong poloidal electric fields due to low conductivity [6], strong $\vec{E} \times \vec{B}$ drifts are responsible for plasma redistribution in the edge. Therefore, understanding current structure in the SOL is quite important. Analysis is performed on the basis of modeling done by the SOLPS-ITER code for different regimes of ASDEX Upgrade (AUG), L and H-modes, singlenull configurations, and for GLOBUS-M, H-modes, both single and double null configurations. Simulation results are compared with probe measurements of the currents at the divertor plates. It is shown that current density distribution at the divertor plates obtained in the modeling is in good agreement with experimental measurements. The role of parallel (poloidal) currents in the formation of a potential maximum/minimum in the vicinity of $X$-point for strongly detached regimes is also analyzed. Parallel currents for low parallel conductivity require poloidal electric fields directed away from $X$-point for normal direction of magnetic field (towards $X$-point for unfavorable direction). In the private flux region (PFR) the poloidal electric field is directed towards (from) the plates for the normal (reversed) direction of the magnetic field. As a result, maximum (minimum) of potential is formed in the $X$-point vicinity causing clockwise $\vec{E} \times \vec{B}$ drifts around the $X$-point.

\section{Pfirsch-Schlüter and thermoelectric currents}

PS current compensates divergence of diamagnetic current ( $\nabla B$ driven current), Fig.1. Its value in the SOL can be obtained in a way similar to derivation for the core from the equation $\nabla \cdot \vec{j}=0$ assuming that diamagnetic current and PS current gives the main contribution. If one assumes that in the SOL above the $X$-point total pressure is constant along magnetic field lines as in the core (which is rather close to reality), then [1] 


$$
j_{\|}=\frac{B_{z}}{B_{x} B}\left(1-\frac{B^{2}}{\left\langle B^{2}\right\rangle}\right) \frac{\partial p}{h_{y} \partial y}+\frac{\text { const }}{b_{x} h_{y} h_{z}} .
$$

Here $x$-poloidal, $y$-radial, $z$-toroidal dimensionless coordinates used in SOLPS simulations [7], $h_{x}, h_{y}, h_{z}$-metric coefficients, $b_{x}=B_{x} / B$, angle brackets correspond to volume average quantity, const is a constant for a given magnetic surface. In contrast to the well-known expression for PS currents in the core, in the SOL there is an arbitrary constant in Eq. (1). In the core this constant is zero. Outside separatrix this constant may be attributed to the thermoelectric current which usually flows from outer to the inner plate. Note that according to Eq. (1) the poloidal component of this current flowing between two flux surfaces is conserved and thus is flowing from one divertor plate to another. We shall define PS current as the first periodic part of the parallel current in Eq. (1)

$$
j_{\|}^{P S}=\frac{B_{z}}{B_{x} B}\left(1-\frac{B^{2}}{\left\langle B^{2}\right\rangle}\right) \frac{\partial p}{h_{y} \partial y} .
$$

In the modeling PS currents in the SOL are well-reproduced, see for example result of AUG L-mode simulation figure 4 in [1]. Fig.2 shows modelled value of parallel current and the parallel current calculated with equation (1) for the ASDEX Upgrade L-mode shot 34685 . Here one can see negative thermoelectric current flowing from the outer to the inner plate and typical PS modulation. Thermoelectric current is caused by the temperature difference between outer and inner plates. Its value therefore depends on the tokamak regime and, in particular, on the degree of detachment. Example is presented in figure 6 of [6], where simulated net poloidal current flowing through the divertor plates is shown as a function of gas puff value which is connected with degree of detachment. The strong decrease of the thermoelectric current through the plates with gas puff corresponds to the temperature decrease at the outer divertor plate.

\section{Plate closing currents}


In addition to the parallel (poloidal) currents discussed above there are currents flowing to (away from) the plates which can be called plate closing currents (PCC). They can be separated into two groups.

First type of PCC closes $\nabla B$ driven currents for normal direction of the magnetic field flowing to PFR below the $X$-point or away from the PFR above the $X$-point for double-null configuration. At the lower outer target this current is directed to the plate (is subtracted from thermoelectric current) at the PFR part of the target and is added to thermoelectric current at the SOL part of the target see schematic Fig. 3. For the first time current flowing to the outer plate in the PFR was obtained in the simulations [1] and was measured at AUG [8].

Second type of PCC is associated with the parallel (poloidal) currents closing radial currents flowing in the SOL above the $X$-point for single-null configuration or between two $X$ points for double-null configuration. According to the analysis [4] a radial current of "neoclassical" nature flows in the SOL. This radial current and associated radial ion flux can determine, as was suggested in [9], SOL width. This assumption was supported by several simulations [10]-[11], [4] where anomalous transport coefficients were strongly reduced in the SOL. Net radial current through the separatrix is zero, since no integral current can flow through the closed flux surface [4], furthermore in the SOL the radial current reaches a maximum and is reduced in the far SOL. Therefore, a pair of parallel (poloidal) currents should close radial current as shown in Fig.4a that is reproduced figure 1 from [4]. The circuit of PCC is shown in figure $4 \mathrm{~b}$. In the experiments these currents could be to a large extent masked by the first type of PCC and the thermoelectric current.

\section{Results of Globus-M modeling}

\subsection{Disconnected double-null configuration}

Here we are presenting some of simulation results obtained with earlier version of SOLPSITER (SOLPS5.2) code for Globus-M tokamak for disconnected double null (DND) configuration in the $\mathrm{H}$-mode for the favorable direction of the magnetic field and comparison with probe measurements at the divertor plates. For details of the simulations see [3], experiment is described in [12]. 
The poloidal current density at the divertor plates is shown in Fig.5a for the Globus-M 34439 shot. Current density is defined as ratio of current to the target with the corresponding sign to the surface of the target. Currents in Fig.5a are also shown in figure 9 of [3]. At the SOL part of the outer plate poloidal current is flowing away from the plate, as one can expect for the thermoelectric current (with exception of the far SOL). The potential here is reduced with respect to the floating potential, which is consistent with the current direction, Fig.5a. The value of current from outer lower target between two separatrixes is 137A. The value of current from the plasma to the lower inner target between two separatrixes is 29A. So the current flowing from the outer plate to the inner plate is not conserved within flux tube as one would expect for the thermoelectric current. Here one has to keep in mind that for conserved current in the spherical tokamak current density at the inner plate should be significantly larger than that at the outer plate due to smaller major radius. The observed reduction of the inner plate current density can be explained by the presence of PCC. At the SOL Strike Point vicinity of the lower outer target PCC is flowing away from the plate, Fig.3, and hence is added to the thermoelectric current. Part of the SOL poloidal current below the $X$-point goes to PFR due to vertical $\nabla B$ driven currents and returns to the outer and inner PFR parts of the plates, where PCC are directed towards the plates. The latter corresponds to the positive peak in Fig. 5a at the PFR part of the outer plate and the negative one at the PFR part of the inner plate. PCC flowing to the plates in the PFR are consistent with the fact, that the plasma potential here is larger than the floating one.

At the outer plate the measured distribution of the poloidal current density and plasma potential in front of the plate, Fig. 5b, are presented [12]. The measurements have been made not in the modelled discharge 34439 but in the similar one. One can see that measurements are in reasonable agreement with the simulation results. It is worth noting that at the inner plate in the simulations (unfortunately no measurements are available here) plasma potential in the units of $T_{e} / e$ is very large, which means that the current to the inner plate is carried by ions while electrons are kept in the plasma by electric field. Distribution of the poloidal currents in the volume from SOLPS-ITER simulation is shown in Fig.6.

Hence PCC of the first type are obtained in the Globus-M tokamak both in the modeling and in the experiment. 


\subsection{Connected double-null configuration}

The second type of PCC is clearly seen in the simulations of Globus-M performed for the connected double-null (CDN) configuration, where contribution from thermoelectric currents should be smaller due to the smaller difference in temperatures between upper and lower plates [5]. Figure 10a of [5] shows electric current to the outer plates of CDN discharge. In the SOL vicinity of Strike Point current is directed from the targets to the plasma for both targets, this is PCC type 2. Further from Strike Point current change sign for the both targets. In the far SOL thermoelectric current can be observed in the simulations - for both targets current has the same value and direction.

\section{Modeling of ASDEX Upgrade $L$ and H-mode shots}

In this section we present results of dedicated modeling comparison with divertor probe measurements for ASDEX Upgrade (AUG) L-mode and H-mode shots, single-null configuration, normal direction of magnetic field. Two L-mode shots, and one H-mode shot has been simulated with SOLPS-ITER code. For L-mode shot N34685 the following parameters were chosen: discharge power $\mathrm{P}=0.7 \mathrm{MW}(\mathrm{Pe}=0.45 \mathrm{MW}, \mathrm{Pi}=0.25 \mathrm{MW}), \mathrm{D}$ fueling rate $2 \cdot 10^{21} \mathrm{~s}^{-1}$, N seeding rate $1 \cdot 10^{18} \mathrm{~s}^{-1}$. For this shot magnetic perturbation coils were switched on, however we assume that this has modest impact on divertor plasma parameters. Our choice of anomalous transport coefficients is presented in Table1. Fitted density and temperature profiles in the outer equatorial midplane are shown in Fig.7. In Fig.8 distributions of probe saturation current and electron temperature at the outer divertor plate are shown. The modelling reproduces well the experimental results except for the far SOL. Large temperature and density decay lengths in the far SOL were observed on many tokamaks and were discussed in the literature. As an example, see AUG experimental data and modeling results (see [13], Figs.2, 5), where both at the equatorial midplane and at the outer divertor plate electron temperature has long tails. As a possible mechanism one can put forward convective transport in the SOL caused by blobs (see [14]), which are generated in the far SOL. Comparison of measured and simulated poloidal currents at the outer divertor plate is shown in upper graph of Fig.9. The experimental data was acquired from seven earthed Langmuir probes located at poloidal intervals of $2.5 \mathrm{~cm}$ along the outer divertor plate in segment 3 of the ASDEX Upgrade vacuum vessel. Each probe has a poloidal extension of $5 \mathrm{~mm}$, hence there was only $20 \%$ coverage of the region spanned by the probes. To get the continuous spatial coverage in the experimental $J_{\text {target }}$ data shown in the upper graph of Fig.9, discharges with slow separatrix excursions $\geq 2 \mathrm{~cm}$ with quasi-constant plasma conditions were selected. One can see that modeling reproduces experimental measuremens reasonably 
well. Current density distribution at the outer plate is similar to that observed in Globus-M, Figs. 5a-5b. Currents at the outer and inner plates are compared in lower graph of Fig.9. We see that at the SOL part of the plate current density at the inner plate is smaller than that at the outer one. In other words, current flowing from the outer plate to the inner plate is not conserved within flux tube, as it should be for thermoelectric current, but partially goes to PFR due to $\nabla B$ drift and then to PFR parts of both plates. Hence part of the current to the plates can be attributed to PCC. Spatial distrubution of the poloidal currents is shown in Fig.10. The main pattern of the poloidal current distribution for AUG is the same as for disconnected double null configuration in Globus-M.

Current density at the outer plate for similar L-mode shot N34458, discharge power $\mathrm{P}=0.5 \mathrm{MW}(\mathrm{Pe}=0.3 \mathrm{MW}, \mathrm{Pi}=0.2 \mathrm{MW}), \mathrm{D}$ fueling rate $1 \cdot 10^{21} \mathrm{~s}^{-1}, \mathrm{~N}$ seeding rate $1 \cdot 10^{18} \mathrm{~s}^{-1}$ is shown in Fig.11. Again reasonable agreement with experiment is observed.

Parameters of the simuated H-mode AUG shot N34873 are: discharge power $\mathrm{P}=2.5$ MW $(\mathrm{Pe}=1.5 \mathrm{MW}, \mathrm{Pi}=1.0 \mathrm{MW})$, D fueling rate $5 \cdot 10^{21} \mathrm{~s}^{-1}, \mathrm{~N}$ seeding rate $5 \cdot 10^{19} \mathrm{~s}^{-1}$. Our choice of anomalous transport coefficients is presented in Table 2. Fitted density and temperature profiles at the outer equatorial midplane are shown in Fig. 12. In Fig.13 ion saturation current and electron temperature at the outer divertor plate are presented. Current density distribution at the outer plate is shown in Fig.14. Here in addition to distribution obtained for the L-mode case, thermoelectric current in combination with PCC type 1, we see additional current to the outer plate at its SOL part. In particular, an additional maximum of current density at the SOL part of the plate, corresponding to current flowing to the plate, is caused by a pair of PCC of type 2. This pair of currents is clearly seen in the 2D plot of poloidal currents, Fig.15. In the far SOL the main contribution is from thermoelectric current.

\section{Role of parallel currents in the formation of a potential extremum in the vicinity of the $X$-point in the detached regime}

In strongly detached regimes in the vicinity of the $X$-point for the normal direction of the magnetic field a potential maximum is observed in the simulations [6]. When the seeding rate is large enough, a cold high radiating region is observed here both in the simulations and in the experiments [15]-[16]. As was first discussed in [6] parallel currents and corresponding parallel (poloidal) electric fields are responsible for formation of the extremum of the potential. Here we demonstrate this using SOLPS-ITER simulations for AUG-type H-mode 5MW shot with 
large $N$ seeding rate. Distribution of the poloidal currents is shown in Fig. 16. One can see that in the vicinity of the $X$-point poloidal currents are directed away from it. Inside and outside the separatrix above the $X$-point PS currents Eq. (2) for the normal direction of the magnetic field flow upwards from the $X$-point. In the PFR, poloidal currents close the vertical $\nabla B$ drift current and are directed towards the plates, i.e. away from the $X$-point. The same is true for currents outside the separatrix but very close to it below $X$-point due to some shift of the plasma pressure maximum towards the far SOL. As was shown in [6] in the detached cases with low temperatures near the $X$-point and low parallel conductivity, the parallel electric field approximately corresponds to Ohm's law $j_{\|}=\sigma_{\|} E_{\|}$because electron pressure gradient and thermal force can be neglected in the parallel momentum balance for electrons. Let us introduce a dimensionless parameter, which demonstrates how good Ohm's law is satisfied:

$$
R=\left|j_{\|}\right| \sigma_{\|} \mid /\left(\left|j_{\|}\right| \sigma_{\|}|+| j_{\|} / \sigma_{\|}-E_{\|}\right)
$$

When $R=1$ the Ohm's law $j_{\|}=\sigma_{\|} E_{\|}$is satisfied, for small $R$ in the regions with high parallel conductivity electric field $\left|E_{\|}\right| \gg>\left|j_{\|} / \sigma_{\|}\right|$and potential is of the order of $T_{e} / e$. As is seen from Fig.17, in the vicinity of the $X$-point parameter $R$ is not far from unity, and, therefore, the parallel electric field has the same direction as parallel current. The poloidal electric field is directed away from the the $X$-point in the core, SOL and PFR, and hence the potential at the $X$ point should have a maximum. The corresponding potential 2D distribution is shown in Fig.18. A potential maximum in the $X$-point vicinity causes $\vec{E} \times \vec{B}$ drifts around the $X$-point in the clockwise direction [6] which leads to redistribution of plasma and impurities. This is especially important in the regimes with a highly radiating $X$-point.

For a reversed magnetic field, poloidal projections of PS currents and poloidal currents in PFR change their direction, and, therefore one would expect a potential minimum near the $X$-point. Such a minimum has been observed in the simulations [17].

\section{Conclusions}


Currents at the plates are the sum of thermoelectric current and plate closing currents (PCC). There are two types of PCC: $a$-closing $\nabla B$ vertical current in the divertor regions, below (above) $X$-point in PFR and SOL, $b$-closing radial current in the SOL. Currents at the plates require deviation of the plasma potential from the floating one - edge modeling should be done taking account of currents and electric fields. Parallel currents for low parallel conductivity require poloidal electric fields directed away from the $X$-point for the normal direction of the magnetic field (towards the $X$-point for the unfavorable direction). As a result, a maximum (minimum) of the potential is formed in the $X$-point vicinity causing clockwise $\vec{E} \times \vec{B}$ drifts around the $X$-point.

\section{Acknowledgements}

This work was supported by the Ministry of science and higher education of Russian Federation in the framework of the state contract in the field of science under project No. 0784-2020-0020 using the Federal Joint Research Center "Material science and characterization in advanced technology" (project RFMEFI62119X0021), including the unique scientific facility "Spherical tokamak Globus-M"

\section{References}

1. V. Rozhansky, E. Kaveeva, S. Voskoboynikov, D. Coster, X. Bonnin, R. Schneider 2003 Nucl. Fusion 43614

2. P.J. Harbour 1988 Contrib. Plasma Phys. 28417

3. E. Vekshina, I. Senichenkov, V. Rozhansky, E. Kaveeva, N. Khromov, G. Kurskiev, M. Patrov and the Globus-M team 2016 Plasma Phys. Control. Fusion 58085007

4. V. Rozhansky, E. Kaveeva, I. Senichenkov and E. Vekshina 2018 Plasma Phys. Control. Fusion 60035001

5. E. Vekshina, V. Rozhansky, E. Kaveeva, I. Senichenkov, N. Khromov 2019 Plasma Phys. Control. Fusion 61125009

6. V. Rozhansky, E. Kaveeva, I. Senichenkov, E. Sytova, I. Veselova, S. Voskoboynikov, D. Coster Contributions to Plasma Physics 2018;58:540-546.

7. R. Scheider, X. Bonnin, D. Coster, H. Kastelevicz, D. Reiter, V. Rozhansky, B. Braams Contrib. Plasma Phys. 200646 N1-3 pp. 3-191 
8. E. Mazzoli. Fluid modelling of electric currents and drifts in the scrape-off layer of ASDEX UPGRADE, 2001, Tesi di Laurea Politechnico di Torino.

9. R. J. Goldston 2012 Nucl. Fusion 52013009

10. E.T. Meyer et al 2016 Plasma Phys. Control. Fusion 58125012

11. E.T. Meyer et al 2017 J. Nucl. Mater. Energy 12973

12. N Khromov et al Technical Physics 2020 in press

13. L. Aho-Mantila et al 2017 Plasma Phys. Control. Fusion 59035003

14. D.Carralero et al 2014 Nucl. Fusion 54123005

15. F Reimold et al 2015 Nucl. Fusion 55033004

16. S Potzel et al 2014 Nucl. Fusion 54013001

17. M Wensing et al 2020 Nucl. Fusion in press

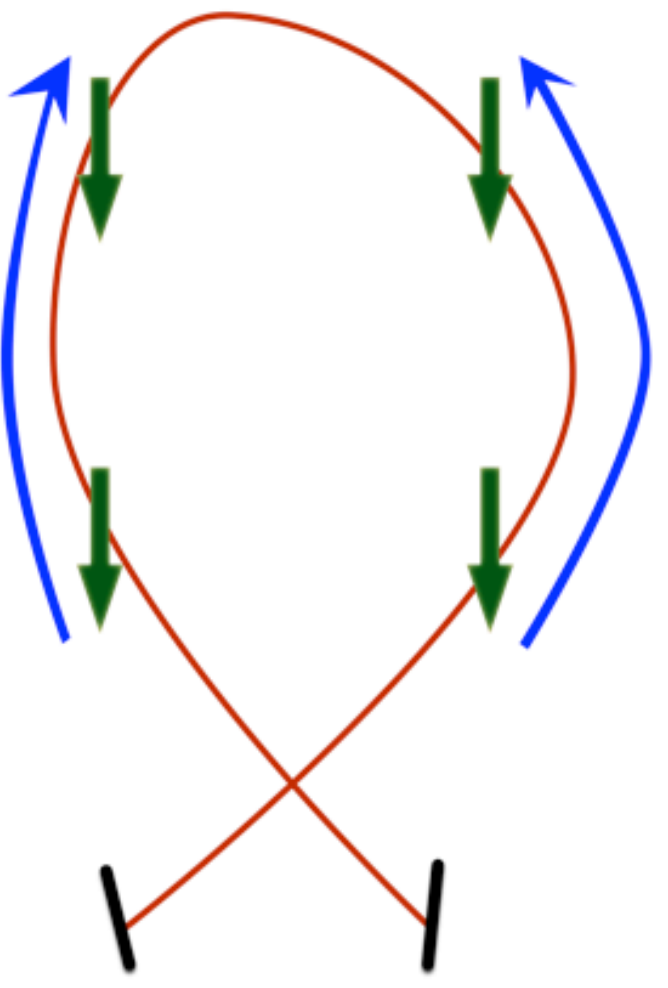


Fig.1. Poloidal components of PS currents closing $\nabla B$ driven currents.

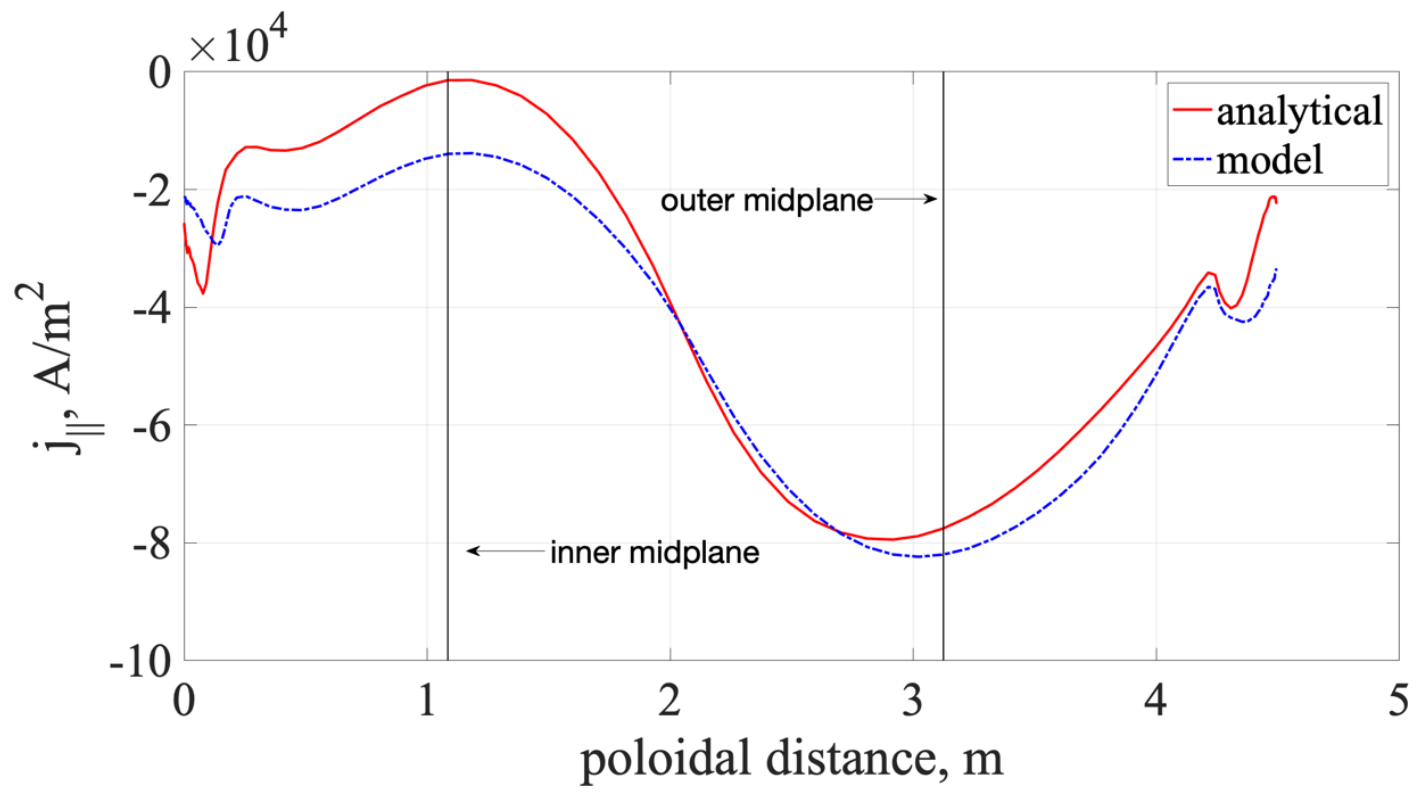

Fig.2. Parallel current in the SOL for the flux surface at the distance $2.4 \mathrm{~mm}$ from the separatrix (at the outer midplane) for AUG L-mode shot 34685. Red line - current, calculated with Eq. (1), constant is chosen to produce best fit with simulations at the top of tokamak; blue dashed line - current calculated in the SOLPS-ITER two-dimensional code. 


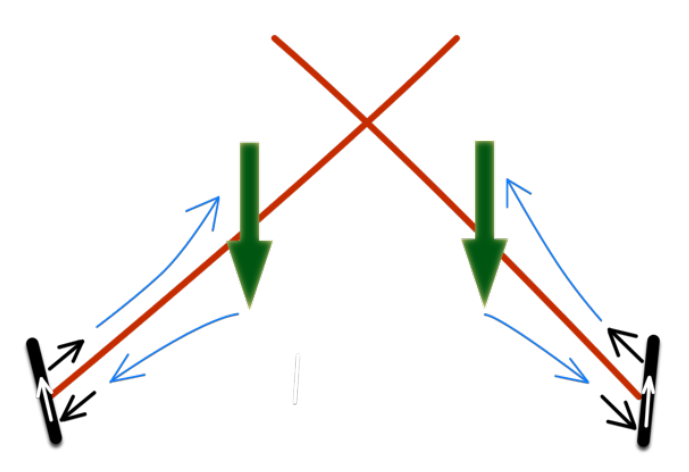

Fig. 3. PCC type I (black arrows) closing $\nabla B$ driven currents (green arrows) below $X$-point. Currents through plasma along magnetic field are shown by blue arrows. Currents through the divertor targets are shown by white arrows. 


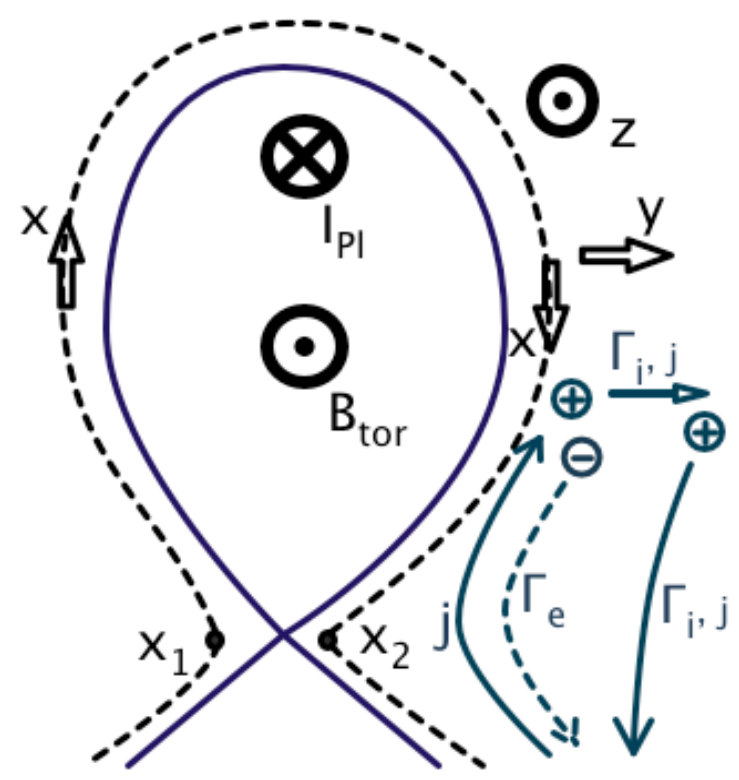

Fig. 4a. Ion flux (solid teal color arrows), electron flux (dashed teal color arrow) and parallel current induced by these fluxes in the SOL. Figure is reproduced from figure 1 of [4]. 


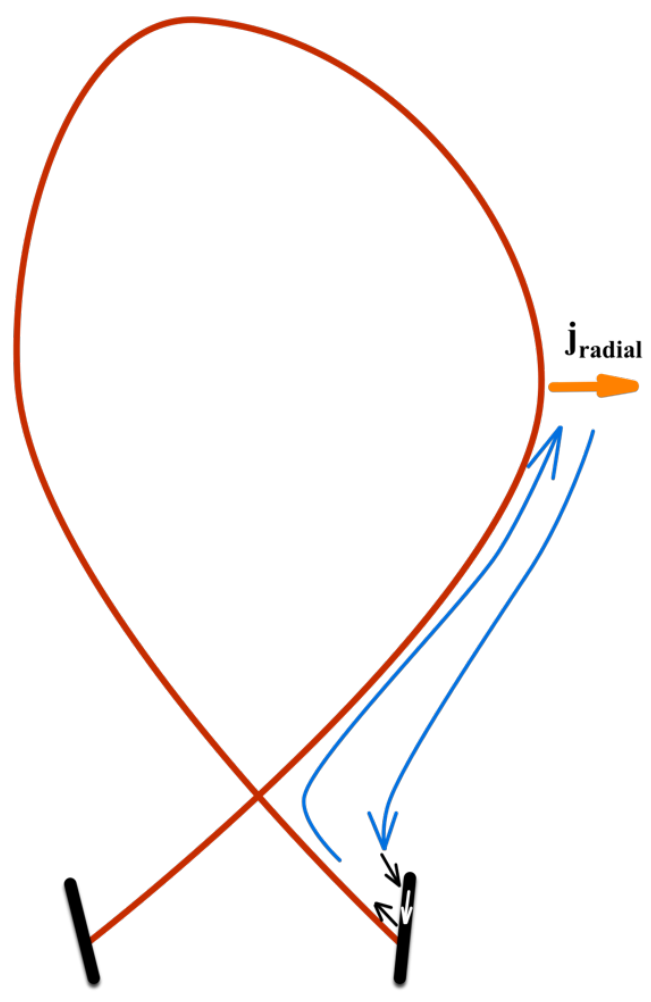

Fig. 4b. Scheme of PCC type 2. Radial current is shown by orange arrow. Poloidal projections of parallel currents are shown by blue arrows. PCC type 2 (black arrows) and current through the target (white arrow) circuit this pair of currents. 


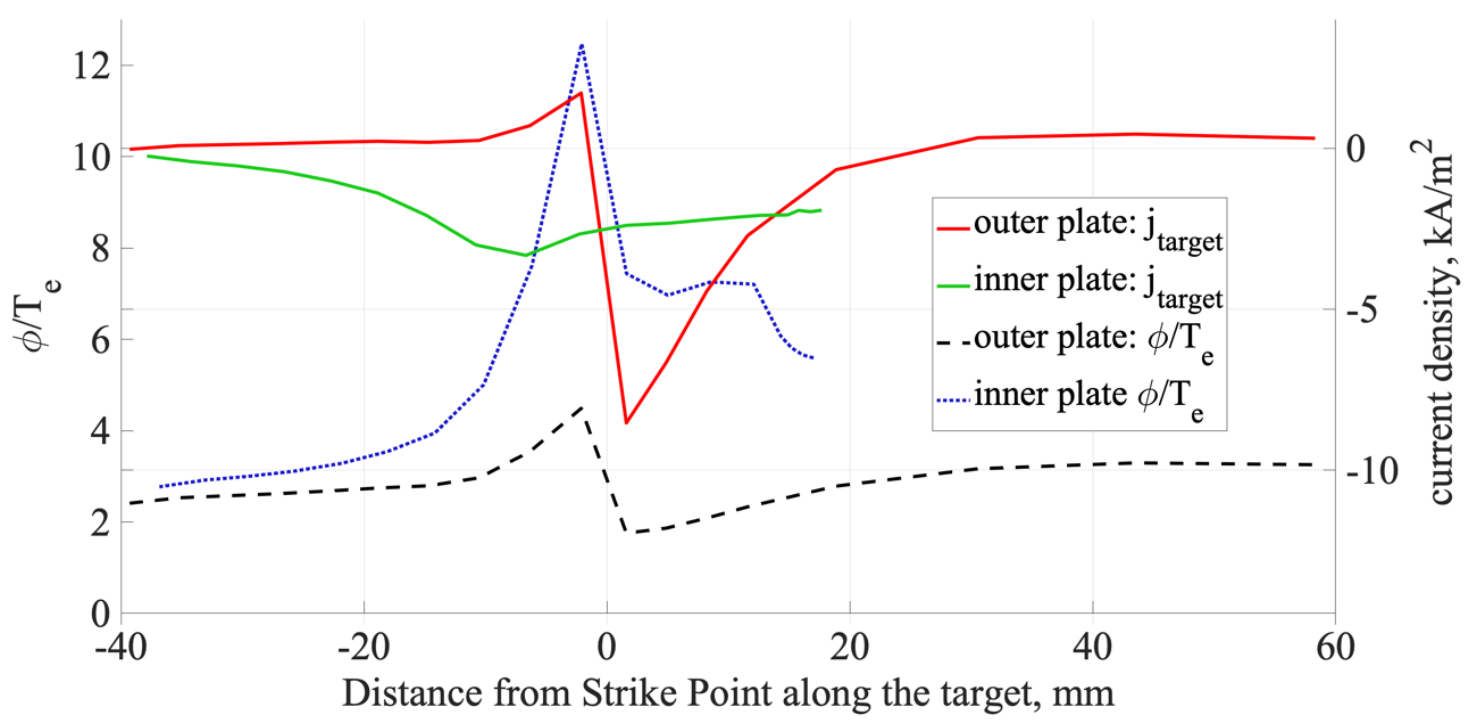

Fig. 5a. Current density at the divertor targets for Globus-M H-mode shot 34439, DND configuration, modeling with SOLPS-ITER. The positive values of $\mathrm{x}$-axis correspond to the SOL part of both targets, negative values of x-axis correspond to the PFR part of both targets. Positive sign of current at the outer plate corresponds to current to the plate, at the inner plate -away from the plate. 


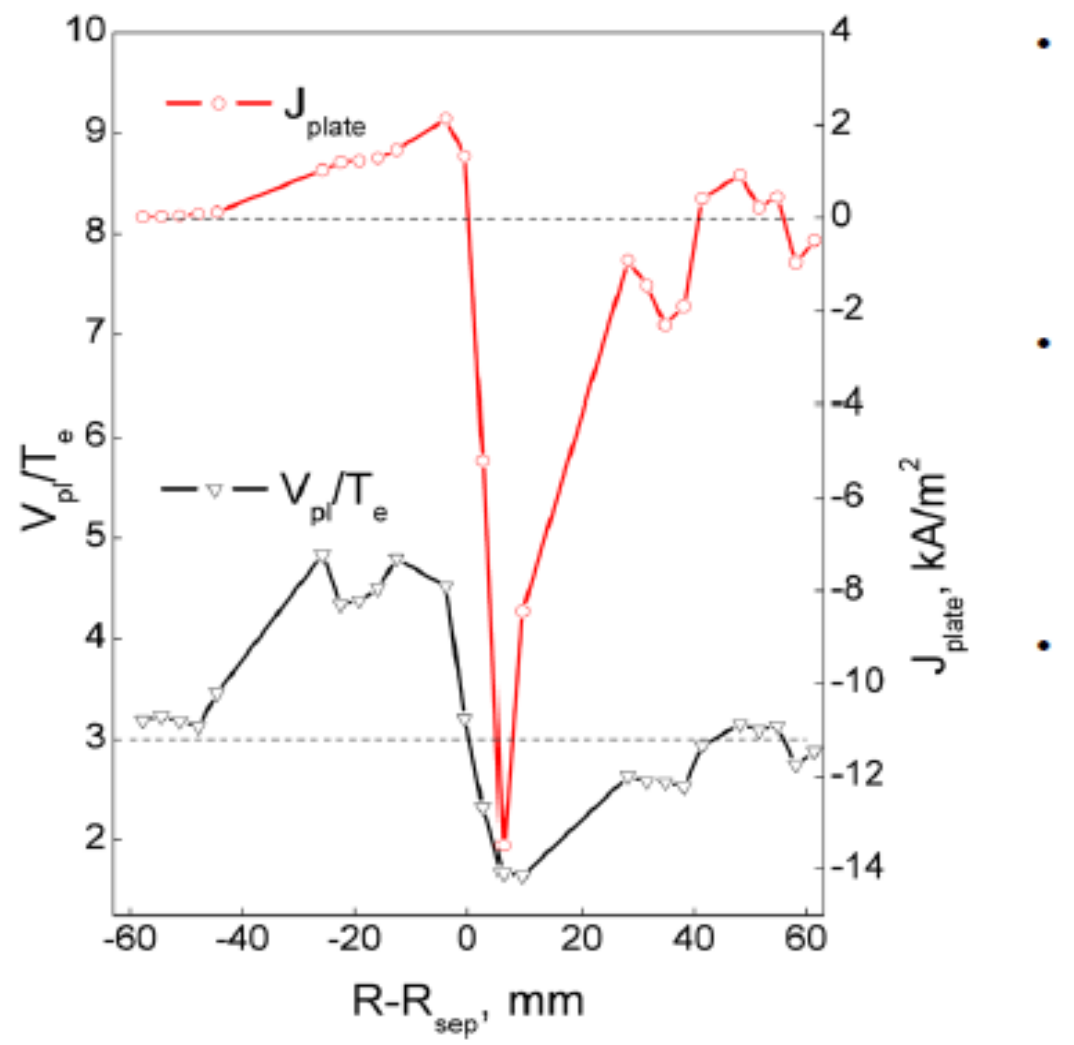

Fig.5b. Current density at the outer divertor target (red curve) for Globus-M H-mode, singlenull configuration, experiment. Positive sign at the outer target corresponds to current from plasma to the plate. Plasma potential in the units of $T_{e} / e$ is shown by black curve. 


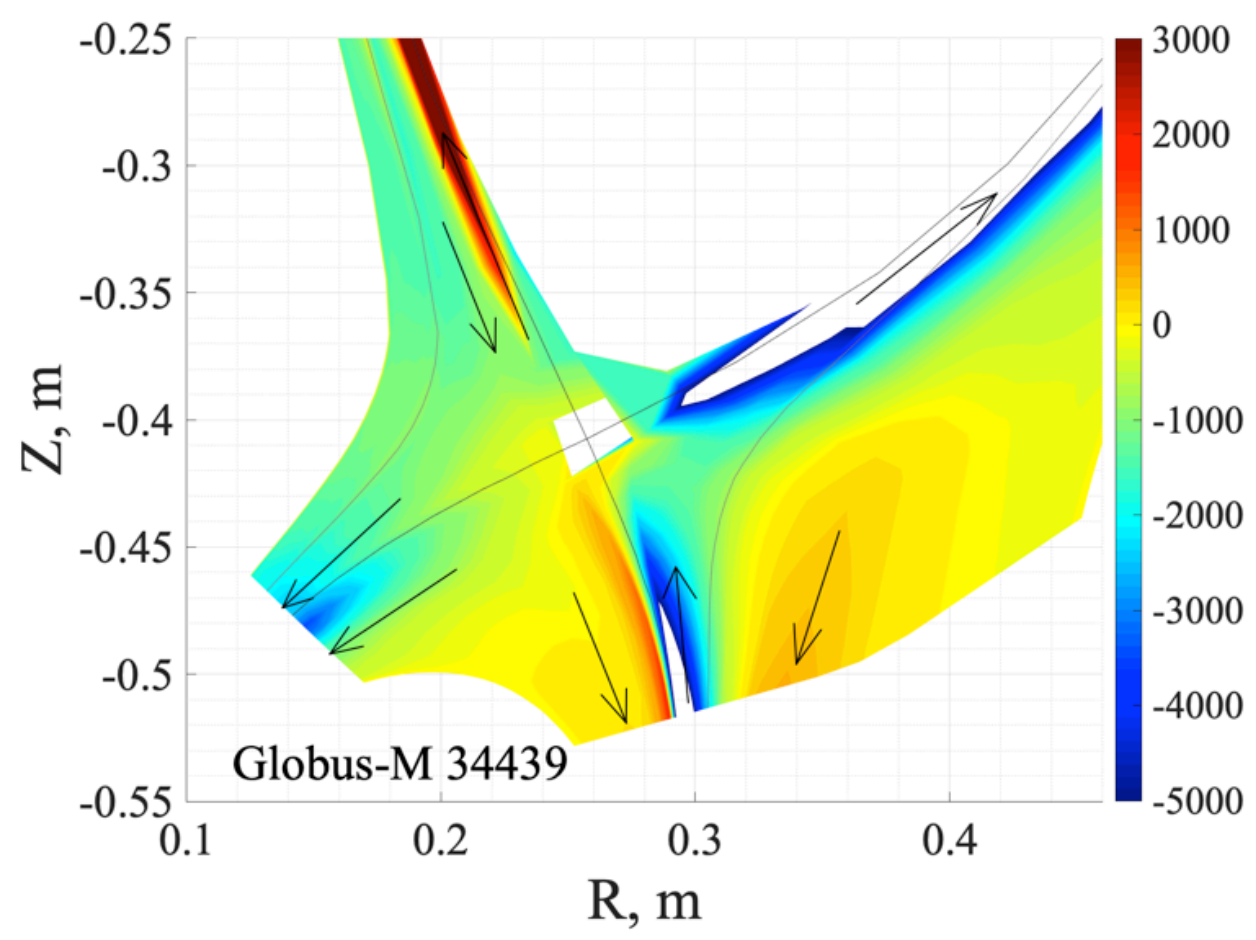

Fig.6. Poloidal current density in Globus-M 34439 shot simulation with SOLPS-ITER.

\begin{tabular}{|c|c|c|c|c|c|}
\hline $\begin{array}{c}\text { The zone } \\
\text { The coeff. }\end{array}$ & Pedestal & $\begin{array}{c}\text { Transport } \\
\text { barrier }\end{array}$ & Near SOL & Far SOL & $\begin{array}{c}\text { Below/above the } \\
\text { X-point }\end{array}$ \\
\hline$D^{A N}, \mathrm{~m}^{2} / \mathrm{s}$ & 0.4 & 0.4 & 0.3 & 1.2 & 1.2 \\
\hline$\chi_{e}^{A N}, \mathrm{~m}^{2} / \mathrm{s}$ & 0.8 & 0.8 & 0.4 & 1.6 & 1.2 \\
\hline$\chi_{i}^{A N}, \mathrm{~m}^{2} / \mathrm{s}$ & 0.8 & 0.8 & 0.4 & 1.6 & 1.2 \\
\hline$u_{\text {pinch }}^{A N}, \mathrm{~m} / \mathrm{s}$ & 0.0 & 0.0 & 0.0 & 0.0 & 0.0 \\
\hline
\end{tabular}

Table 1. Transport coefficients for L-mode shot N34685. 

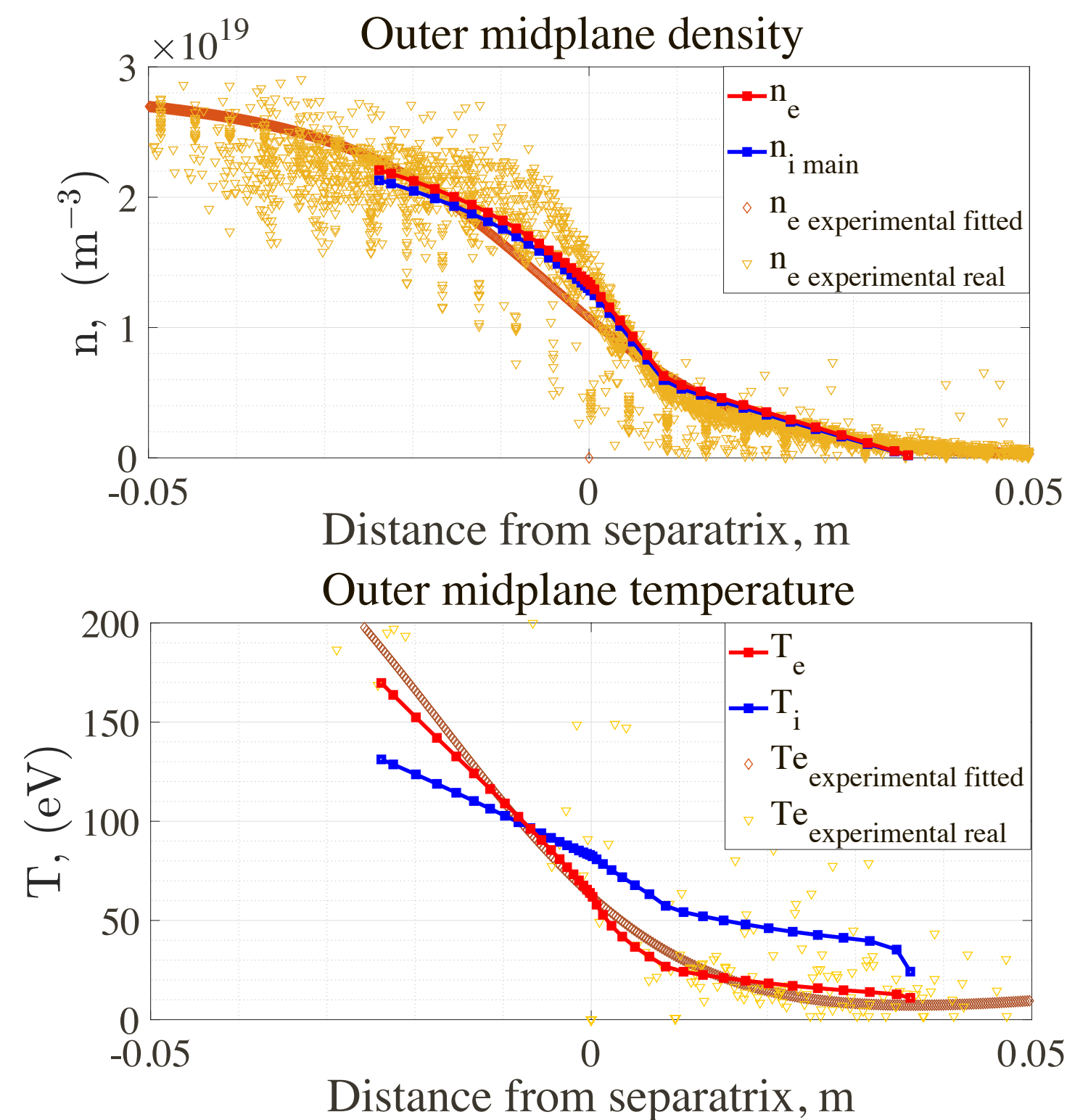

Fig.7. Density and temperature profiles in the outer midplane for L-mode shot N34685. Upper graph - calculated profiles of electron and main ion densities and corresponding experimental data. Here and below curve corresponds to fitted experimental data, brown one-raw experimental data. Lower graph - calculated profiles of electron and ion temperatures and corresponding experimental data. 


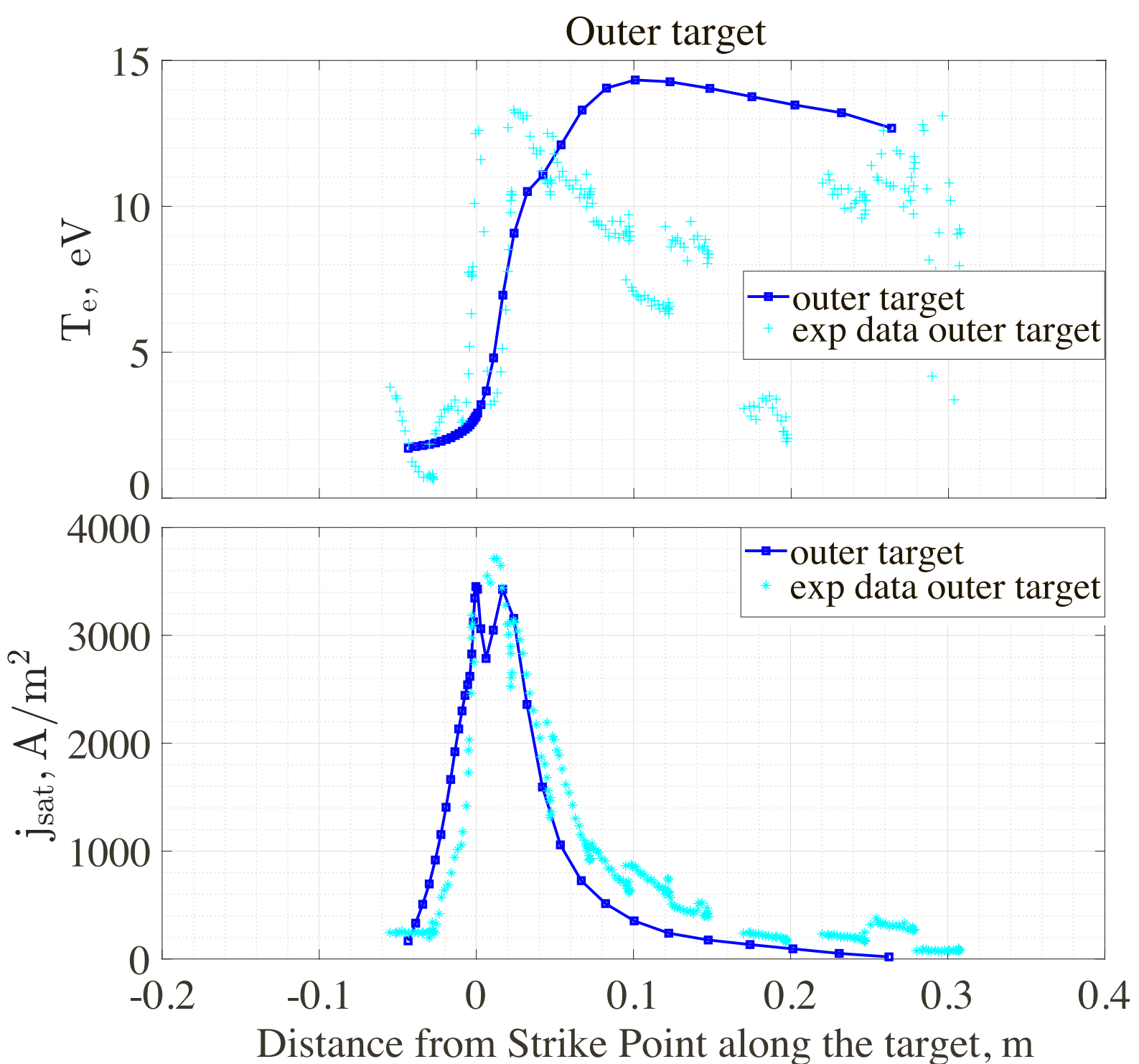

Fig.8. Ion saturation current and electron temperature profiles at the outer target for L-mode shot N34685. Upper graph - calculated ion saturation current profile and corresponding experimental data. Lower graph - calculated electron temperature at the outer divertor target and corresponding experimental data. 

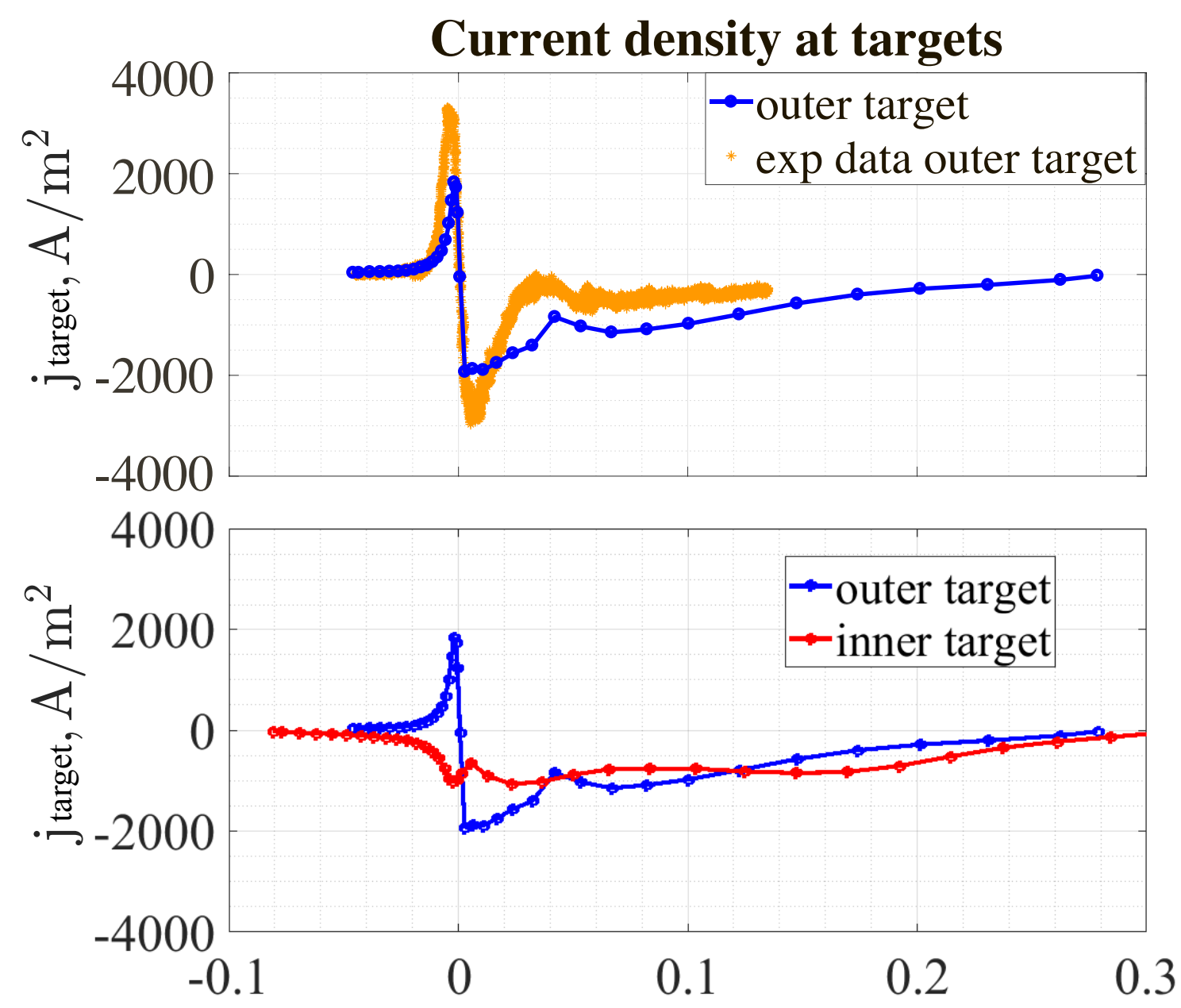

Distance from Strike Point along the target, $\mathrm{m}$

Fig.9. Current at the targets of AUG L-mode shot N34685. Upper graph - calculated current density and corresponding measurements at the outer target. Lower graph - calculated current density at the outer and inner targets. 


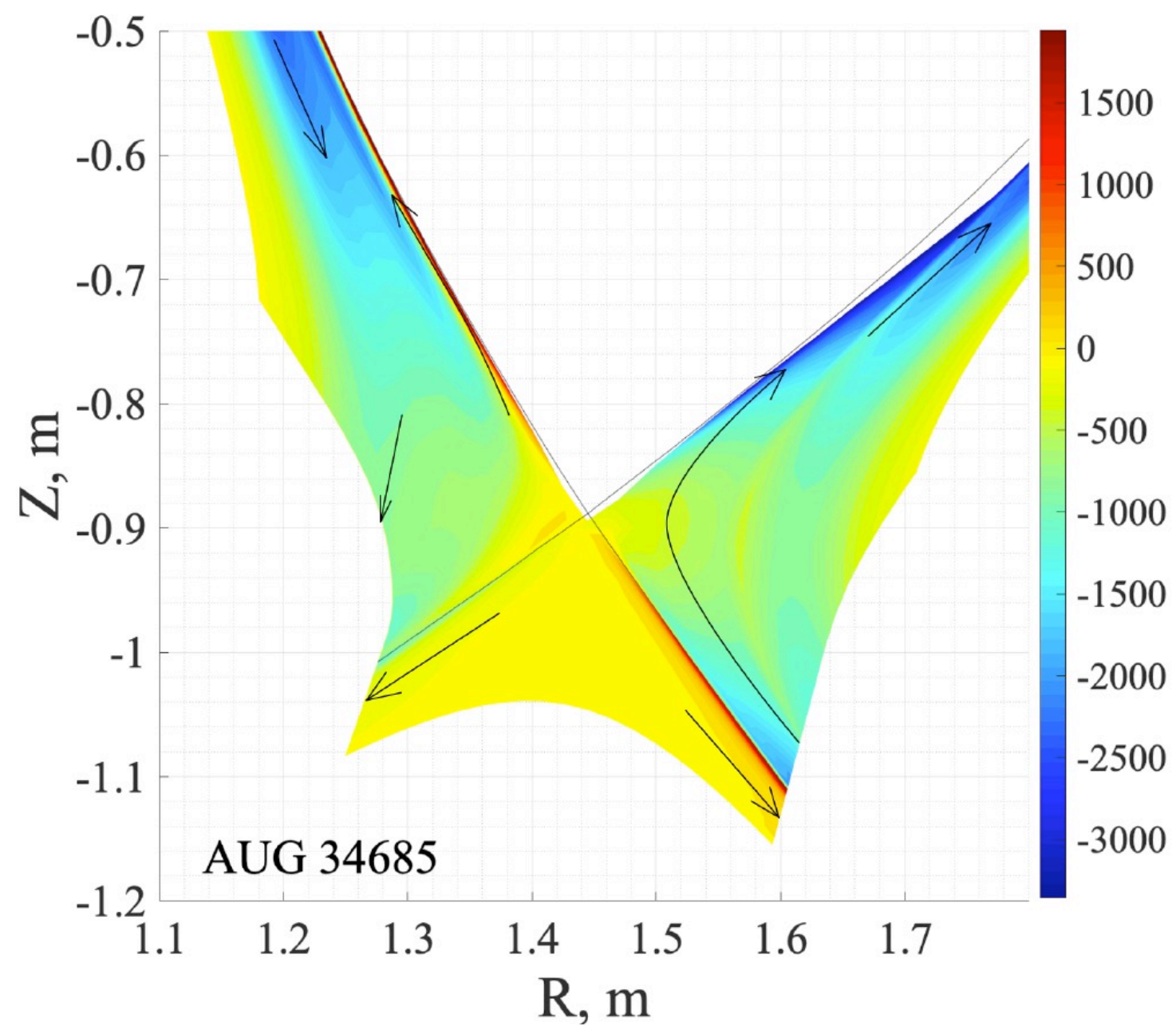

Fig.10. Poloidal current density for L-mode shot N34685. 


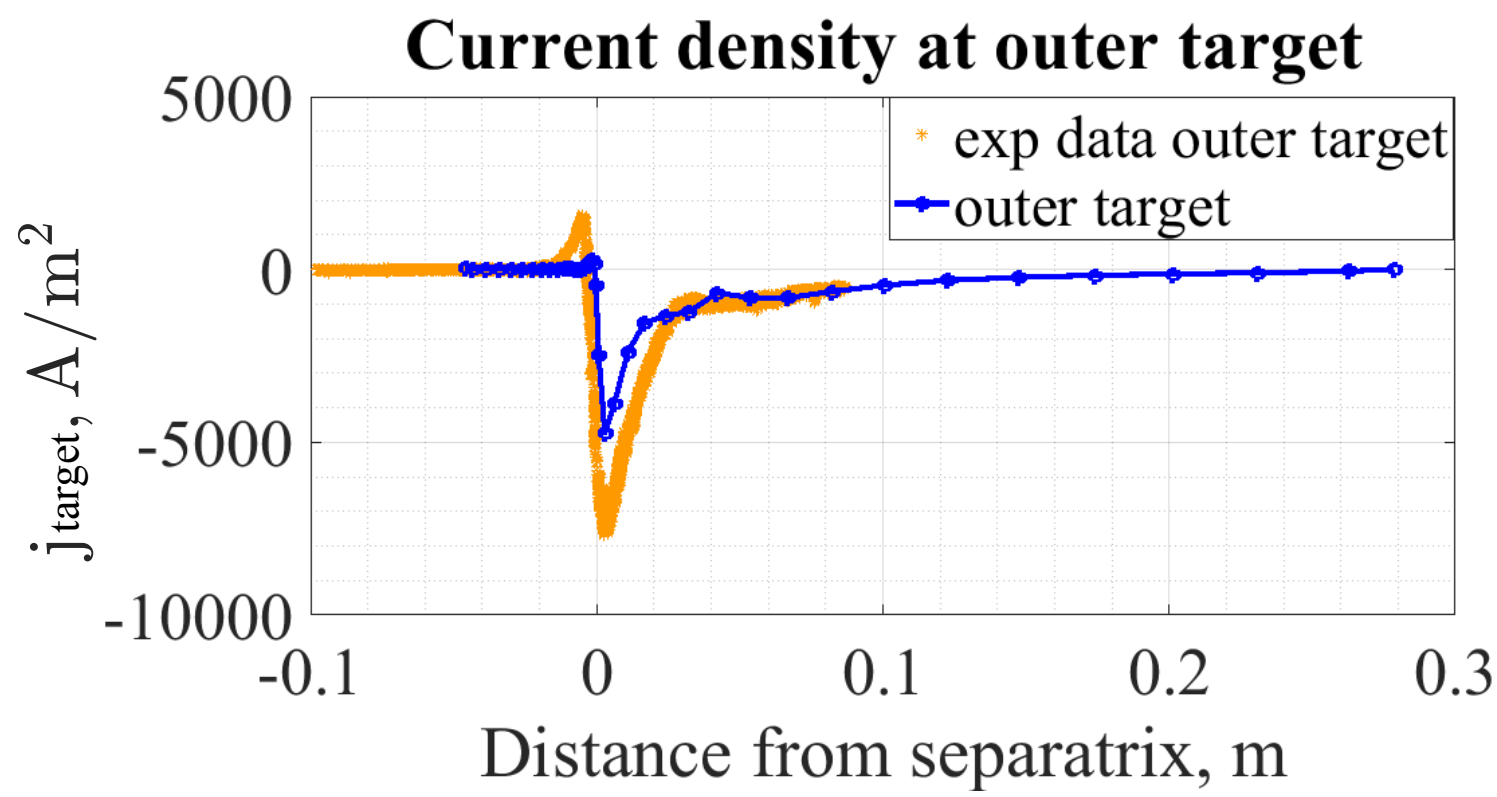

Fig.11. Calculated current density and corresponding measurements at the outer target of AUG L-mode shot N34458.

Table 2.

\begin{tabular}{|c|c|c|c|c|c|}
\hline $\begin{array}{c}\text { The zone } \\
\text { The coeff. }\end{array}$ & Pedestal & $\begin{array}{c}\text { Transport } \\
\text { barrier }\end{array}$ & Near SOL & Far SOL & $\begin{array}{c}\text { Below/above the } \\
\text { X-point }\end{array}$ \\
\hline$D^{A N}, \mathrm{~m}^{2} / \mathrm{s}$ & 0.5 & 0.05 & 0.05 & 1.5 & 1.5 \\
\hline$\chi_{e}^{A N}, \mathrm{~m}^{2} / \mathrm{s}$ & 0.9 & 0.45 & 0.36 & 1.44 & 1.44 \\
\hline$\chi_{i}^{A N}, \mathrm{~m}^{2} / \mathrm{s}$ & 0.9 & 0.2 & 0.2 & 1.44 & 1.44 \\
\hline$u_{\text {pinch }}^{A N}, \mathrm{~m} / \mathrm{s}$ & 0.0 & 0.0 & 0.0 & 0.0 & 0.0 \\
\hline
\end{tabular}

Table 2. Transport coefficients for H-mode shot N34873. 


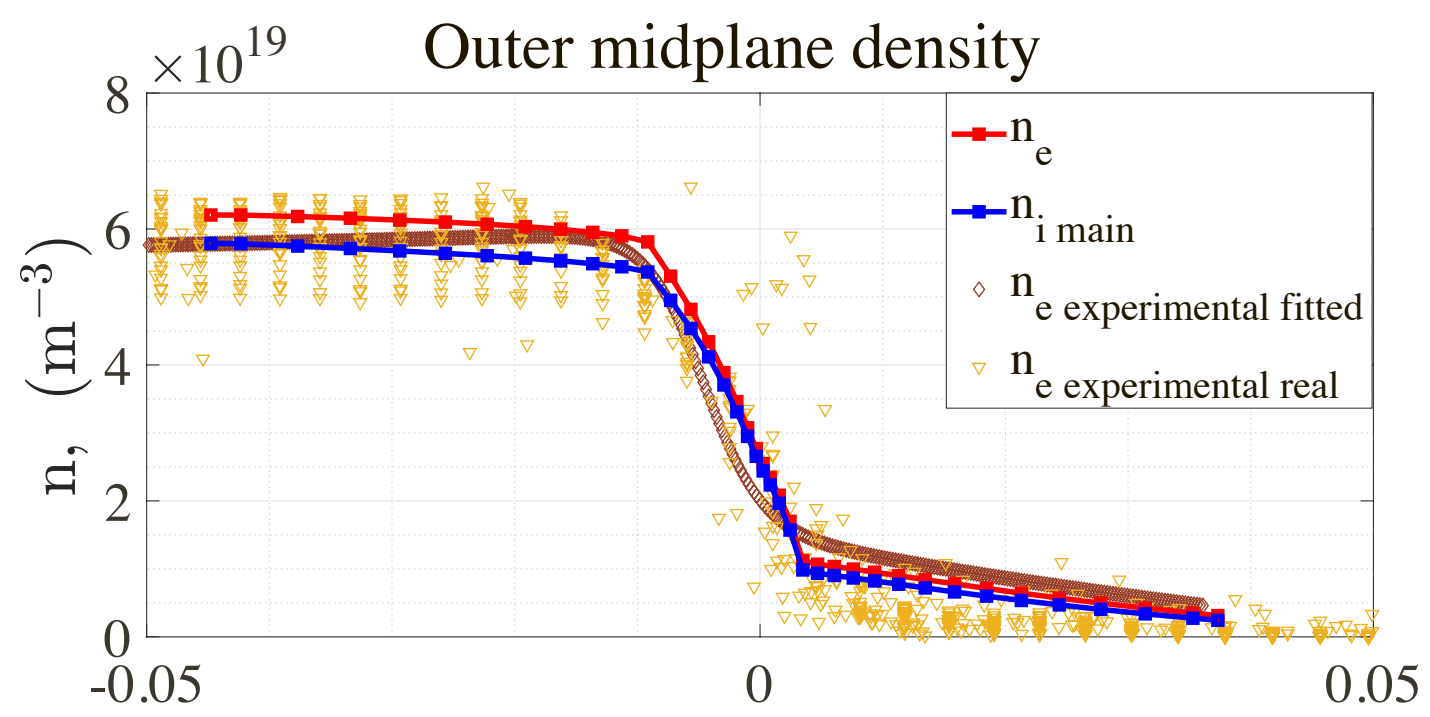

Distance from separatrix, $m$

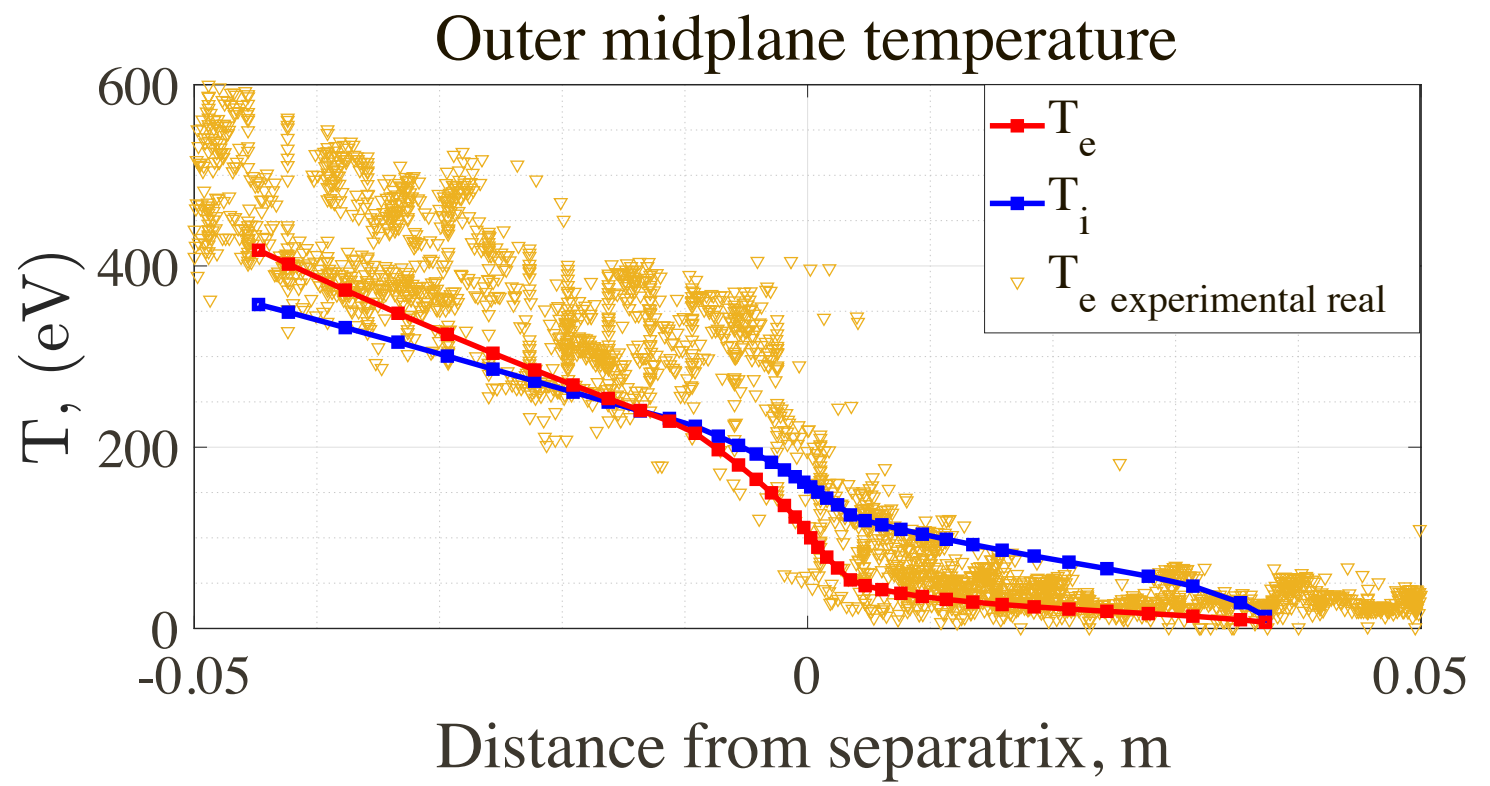

Fig.12. Density and temperature profiles in the outer midplane for H-mode shot N34873. Upper graph - calculated profiles of electron and main ion densities at the outer midplane and corresponding experimental data. Lower graph - calculated profiles of electron and ion temperatures at the outer midplane and corresponding experimental data. 

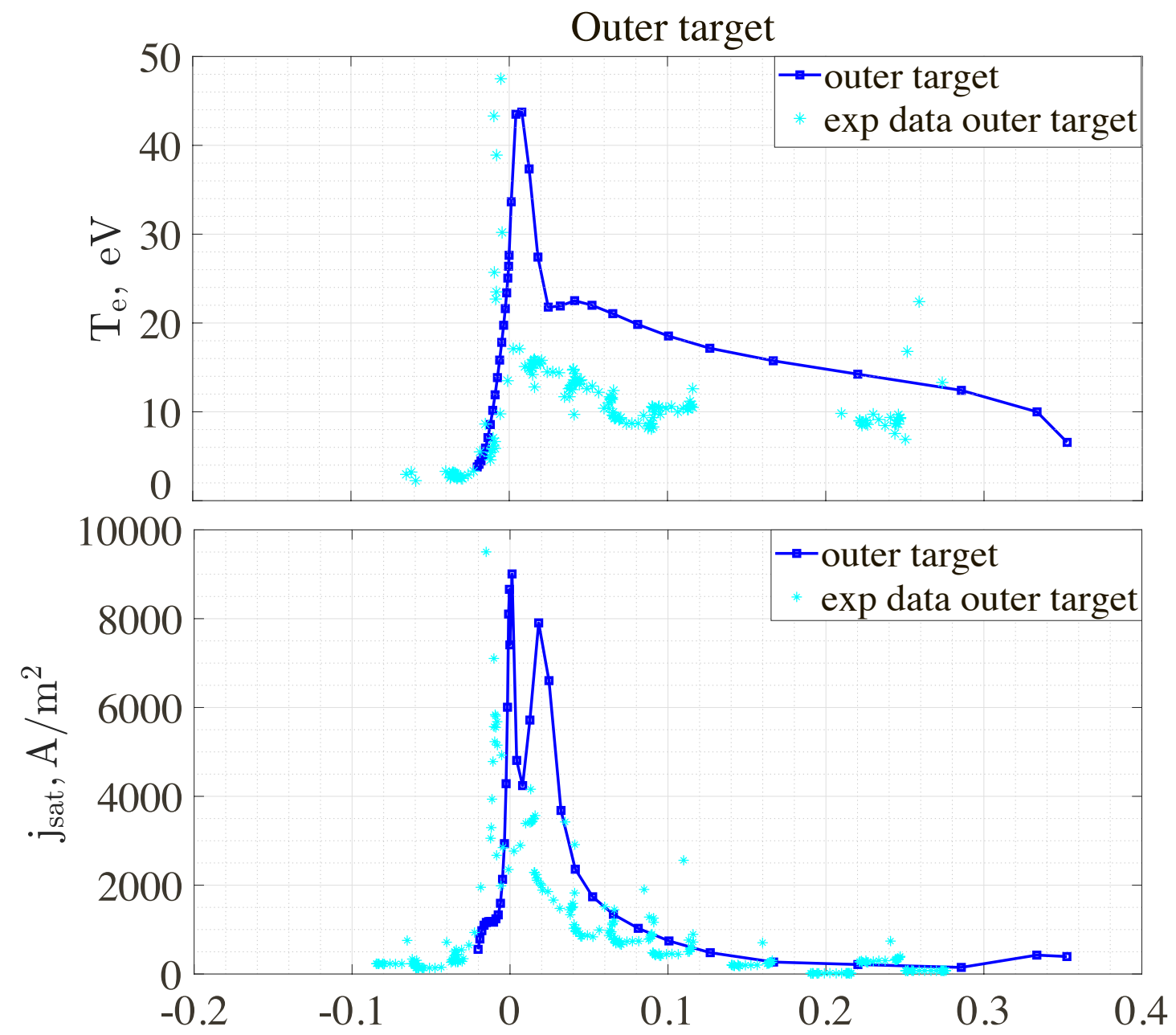

Distance from Strike Point along the target, $\mathrm{m}$

Fig.13. Electron temperature and ion saturation current profiles at the outer target for H-mode shot N34873. Upper graph - calculated ion electron temperature profile and corresponding experimental data. Lower graph - calculated ion saturation current at the outer divertor plate and corresponding experimental data. 


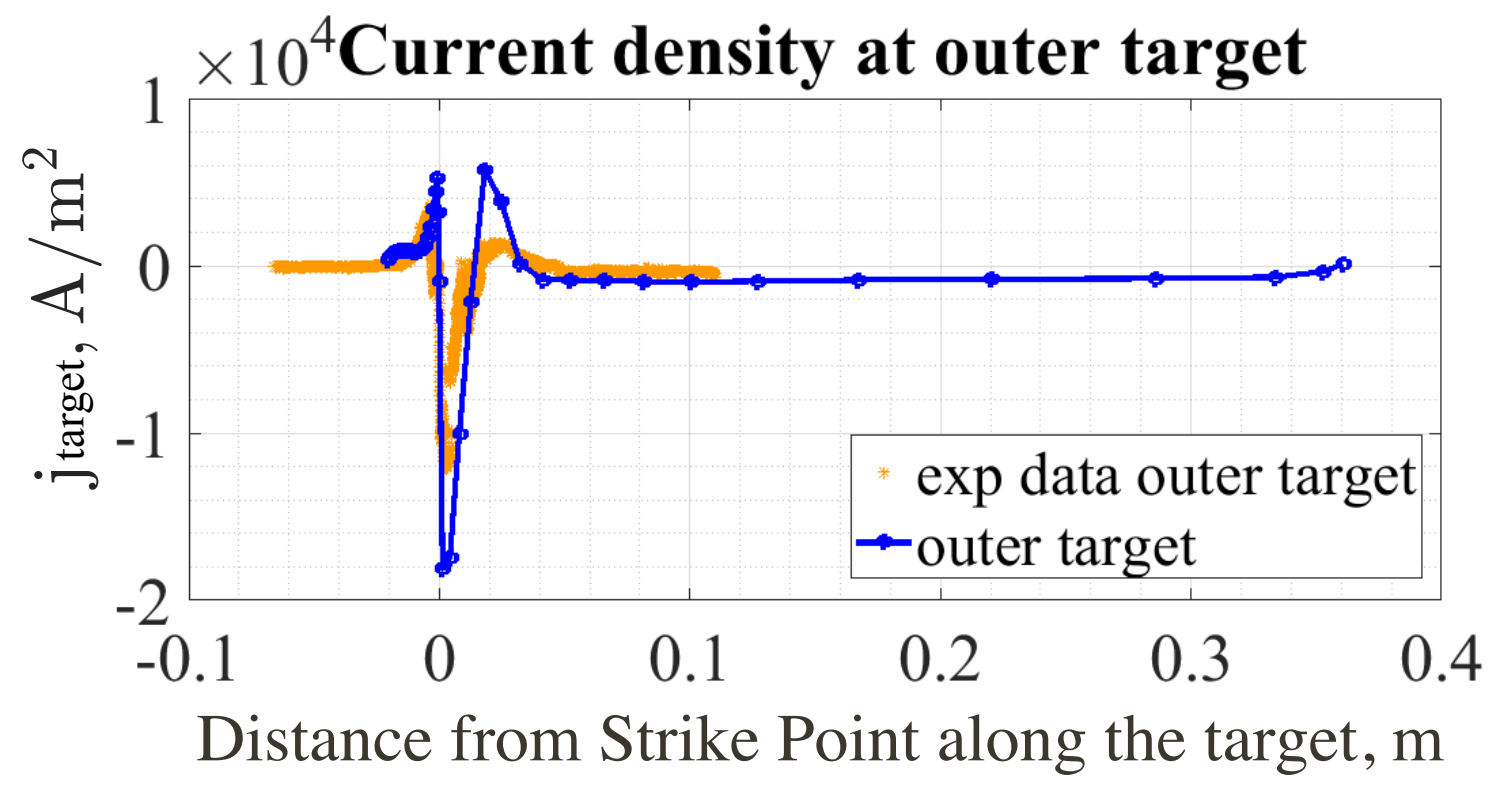

Fig. 14. Calculated current density and corresponding measurements at the outer target of $\mathrm{H}$ mode shot N34873. 


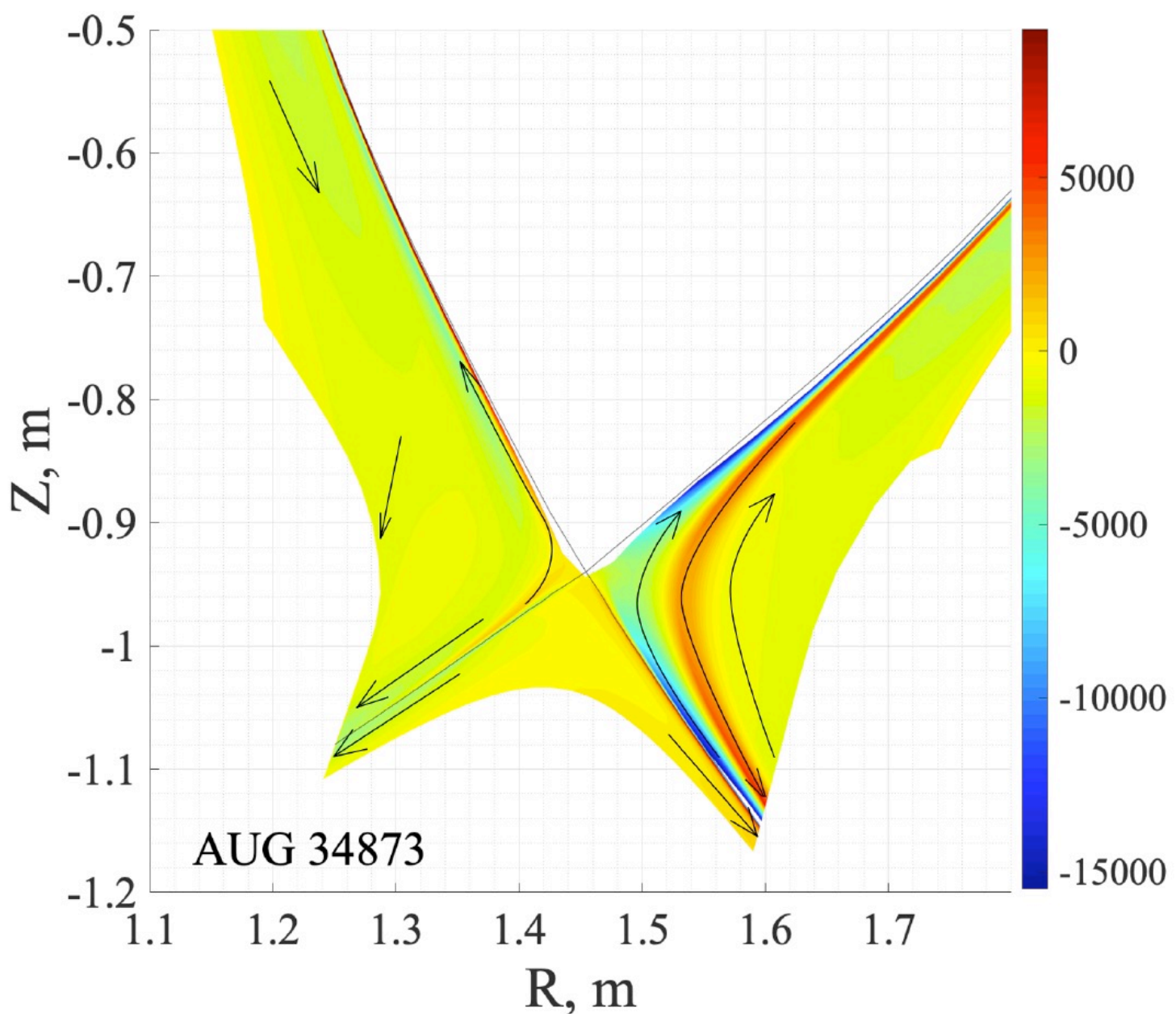

Fig.15. Poloidal current density for H-mode shot N34873. 


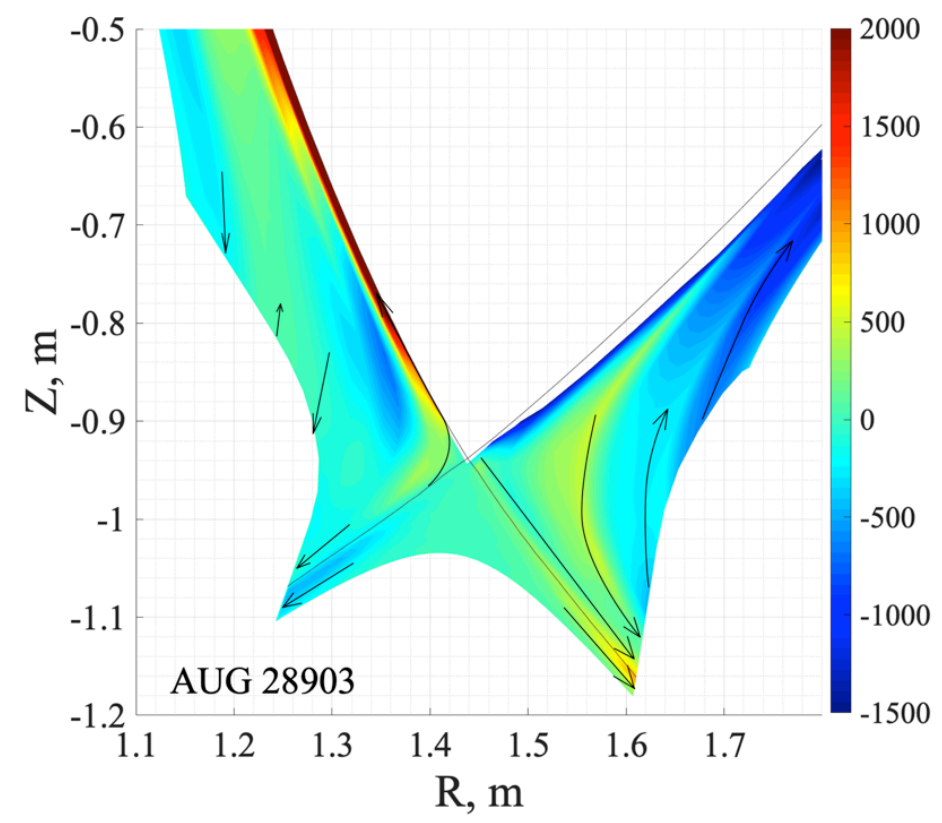

Fig.16. Poloidal current density for H-mode in the detached regime.

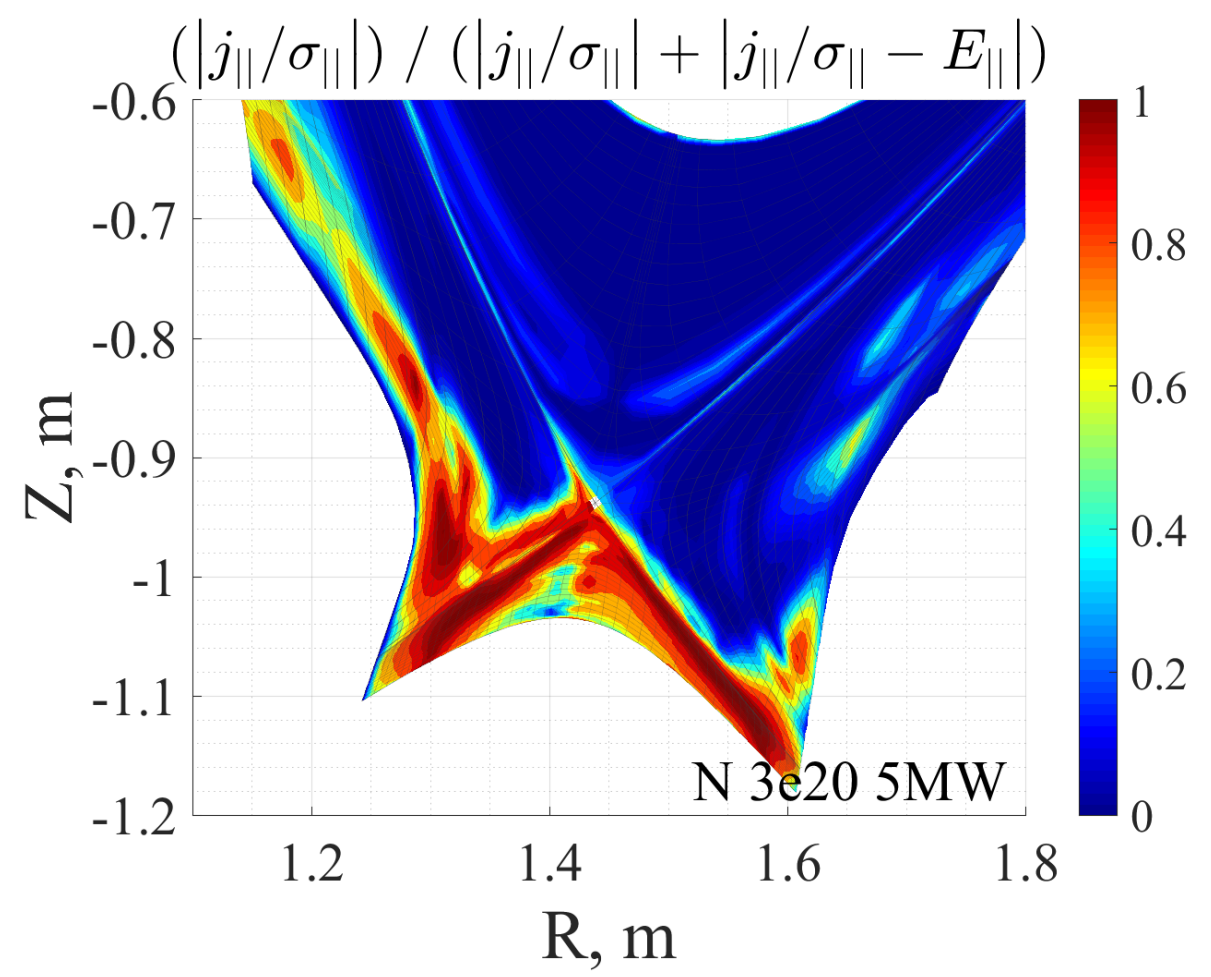

Fig.17. Parameter $R$, which demonstrates how well Ohm's law is satisfied. 


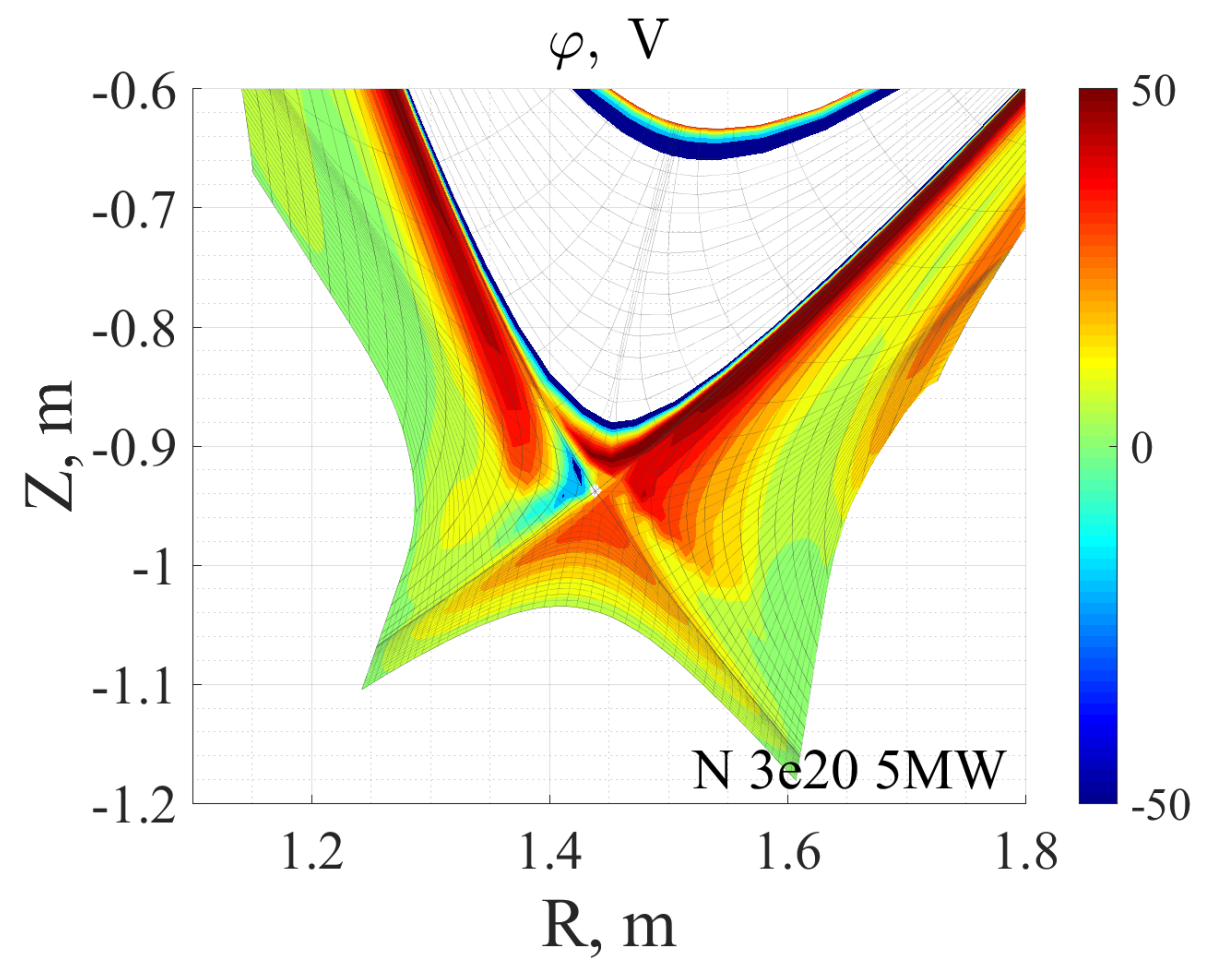

Fig.18. Potential distribution for H-mode in the detached regime. 